\title{
Quantitative next-generation sequencing-based analysis indicates progressive accumulation of microsatellite instability between atypical hyperplasia/endometrial intraepithelial neoplasia and paired endometrioid endometrial carcinoma
}

\author{
David B. Chapel ${ }^{1} \cdot$ Sushant A. Patil ${ }^{1} \cdot$ Andrei Plagov $^{1} \cdot$ Rutika Puranik $^{1} \cdot$ Anastasiya Mendybaeva $^{1} \cdot$ \\ George Steinhardt ${ }^{1} \cdot$ Pankhuri Wanjari $^{1} \cdot$ Ricardo R. Lastra $^{1} \cdot$ Sabah Kadri ${ }^{1}$ Jeremy P. Segal ${ }^{1}$ • \\ Lauren L. Ritterhouse $\mathbb{C}^{1}$
}

Received: 13 March 2019 / Revised: 3 May 2019 / Accepted: 4 May 2019 / Published online: 11 June 2019

(c) United States \& Canadian Academy of Pathology 2019

\begin{abstract}
Atypical hyperplasia/endometrial intraepithelial neoplasia is an accepted precursor to endometrioid-type endometrial carcinoma. Mismatch repair-deficient endometrial carcinomas are also known to be a biologically and clinically distinct subset of tumors. However, the development of microsatellite instability in endometrial carcinogenesis has not yet been evaluated by novel nextgeneration sequencing-based methods. We examined 17 mismatch repair-deficient endometrioid endometrial carcinomas and their paired atypical hyperplasia/endometrial intraepithelial neoplasia precursors using a next-generation sequencing panel with quantitative microsatellite instability detection at 336 loci. Findings were compared to histological features, polymerase chain reaction-based microsatellite instability testing, immunohistochemical expression of mismatch repair proteins, and tumor mutational burden calculations. All 17 endometrial carcinomas and 8/17 atypical hyperplasia/endometrial intraepithelial neoplasia showed microsatellite instability by next-generation sequencing-based testing. Endometrial carcinoma specimens showed significantly more unstable microsatellite loci than paired atypical hyperplasia/endometrial intraepithelial neoplasia (mean: $40.0 \%$ vs 19.9 unstable loci, respectively). Out of nine microsatellite-stable atypical hyperplasia/endometrial intraepithelial neoplasia specimens, four showed mismatch repair loss by immunohistochemistry. All atypical hyperplasia/ endometrial intraepithelial neoplasia and endometrial carcinoma specimens with microsatellite instability were also mismatch repair-deficient by immunohistochemistry. Tumor mutational burden was significantly greater in endometrial carcinoma than in paired atypical hyperplasia/endometrial intraepithelial neoplasia specimens, and tumor mutational burden was significantly correlated with percent unstable microsatellite loci. Paired atypical hyperplasia/endometrial intraepithelial neoplasia and endometrial carcinoma specimens show progressive accumulation of unstable microsatellite loci following loss of mismatch repair protein expression. Comprehensive next-generation sequencing-based testing of endometrial carcinomas offers new insights into endometrial carcinogenesis and opportunities for improved tumor surveillance, diagnosis, and management.
\end{abstract}

This study was presented at the 108th annual meeting of the United States and Canadian Academy of Pathology in National Harbor, Maryland, USA, where it was awarded a Stowell-Orbison Certificate of Merit. This work was awarded first prize in the 2018 Resident Research Competition of the Chicago Pathology Society.

Supplementary information The online version of this article (https:// doi.org/10.1038/s41379-019-0298-5) contains supplementary material, which is available to authorized users.

Lauren L. Ritterhouse

lritterhouse@partners.org

1 Department of Pathology, University of Chicago, 5841S. Maryland Ave, Chicago, IL, USA

\section{Introduction}

Endometrial carcinoma is the most common cancer of the female reproductive tract in the United States, and the fourth most common cancer in American women overall, with nearly 62,000 new cases diagnosed annually [1]. The endometrioid histotype of endometrial carcinoma classically arises in perimenopausal and early postmenopausal women, in the setting of increased estrogen exposure [2]. Endometrioid endometrial carcinoma develops from clonal proliferations of architecturally crowded, cytologically atypical endometrial glands [3-10], termed "atypical 
hyperplasia/endometrial intraepithelial neoplasia" in the 2014 World Health Organization Classification of Tumours of the Female Reproductive Organs [11].

Approximately one quarter of endometrioid endometrial carcinomas exhibit loss of function of one or more of the mismatch repair proteins MLH1, MSH2, MSH6, or PMS2, resulting in the accelerated accrual of lengthaffecting errors in homopolymer tracts (repetitive stretches of the same nucleotide), resulting in a molecular signature termed microsatellite instability [12-14]. Loss of mismatch repair function has been previously shown to be a relatively early molecular alteration in a subset of endometrial carcinomas $[15,16]$. Early studies showed that $M L H 1$ promoter hypermethylation can occur in the absence of an established microsatellite instability molecular signature, and that endometrial carcinoma shows microsatellite instability when associated with microsatellite-unstable atypical hyperplasia/endometrial intraepithelial neoplasia $[15,16]$. In contrast, microsatellite-unstable endometrial carcinoma was associated with microsatellite-stable atypical hyperplasia/ endometrial intraepithelial neoplasia in approximately half of cases [7, 9, 15, 16]. Larger, more recent studies suggest high concordance of mismatch repair protein expression between paired atypical hyperplasia/endometrial intraepithelial neoplasia and endometrial carcinoma specimens $[17,18]$. Although some data suggest that mismatch repair immunohistochemistry performed on specimens containing only atypical hyperplasia/endometrial intraepithelial neoplasia may accurately detect Lynch syndrome, sporadic mismatch repair deficiency may be underreported by this approach, and routine mismatch repair testing on atypical hyperplasia/endometrial intraepithelial neoplasia specimens is not currently a widespread clinical practice $[7,9,18]$.

Clinically, mismatch repair-deficient endometrial carcinoma arising in patients with Lynch syndrome $[19,20]$ must be distinguished from sporadic mismatch repairdeficient carcinomas, which result most commonly from silencing of $\mathrm{MLHI}$ through promoter hypermethylation [18], or alternately through somatic mutations in mismatch repair protein genes. Screening of endometrial carcinomas for Lynch syndrome begins either with immunohistochemistry to detect loss of mismatch repair protein expression in tumor cells, or with polymerase chain reaction-based microsatellite instability testing [21]. An abnormal screening result prompts additional testing to identify or rule out a germline mutation, as recently reviewed [17, 21].

Mismatch repair immunohistochemistry is currently the favored tool for mismatch repair testing, given its cost effectiveness, broad availability, and relative ease of interpretation. In contrast, polymerase chain reaction-based microsatellite instability testing relies on comparison of microsatellite loci in tumor DNA to paired non-tumor DNA from the patient, to detect microsatellite length-affecting alterations in the tumor. Widely adopted sets of microsatellites used for polymerase chain reaction-based microsatellite instability analysis have typically included 5-10 loci, with tumors classified as microsatellite-stable, microsatellite instability-low, and microsatellite instability-high, depending on the number of loci showing microsatellite instability. Polymerase chain reaction-based microsatellite instability calling assays are largely designed to test colorectal cancers, and may produce only subtle shifts in endometrial cancers [22]. Nonetheless, they can be used to detect microsatellite instability in endometrial carcinoma $[23,24]$ and can play an important role in the workup of tumors with ambiguous results on mismatch repair immunohistochemistry.

Recently, novel next-generation sequencing-based methods have been developed for detecting and quantifying unstable microsatellite loci and classifying tumor microsatellite stability [25-28]. Like polymerase chain reactionbased microsatellite instability methods, next-generation sequencing-based microsatellite instability calling tools, compare the number and proportion of discrete microsatellite read lengths in tumor and normal tissue. But in contrast to polymerase chain reaction-based methods, nextgeneration sequencing-based microsatellite instability tools have the ability to evaluate hundreds of microsatellite loci, permitting comparison of tumor microsatellites to a population microsatellite profile derived from a large number of non-tumor specimens and obviating the need for paired normal patient DNA. In addition, although next-generation sequencing-based microsatellite instability calling methods also employ tumor-specific cutoffs [29], analysis of hundreds of microsatellites removes much of the bias inherent to the small microsatellite sets used in polymerase chain reaction-based testing designed for colorectal cancer.

Mismatch repair immunohistochemistry and microsatellite instability testing have also taken on a predictive role in immunotherapy eligibility [30, 31]. On 23 May 2017, the Food and Drug Administration granted their first tissue/site-agnostic approval, with an accelerated approval for pembrolizumab immunotherapy for patients with mismatch repair-deficient or microsatellite instability-high solid tumors that are unresectable or metastatic, have progressed after prior therapy, and lack alternate treatment options [32]. Furthermore, analyses of the Cancer Genome Atlas data have classified mismatch repair-deficient endometrial carcinomas as a discrete prognostic subgroup which led to a novel molecular model for classification of endometrial carcinoma $[12,13]$. Finally, next-generation sequencing results can 
also be used to calculate tumor mutational burden, a holistic measure of accumulated mutations with a growing role in clinical management [33].

Utilizing an in-house developed next-generation sequencing panel incorporating quantitative microsatellite instability detection across 336 loci, we designed a retrospective analysis of endometrioid endometrial carcinoma and their paired atypical hyperplasia/endometrial intraepithelial neoplasia. In addition to evaluating tumors' clinicopathologic features and somatic mutations, this study seeks to better clarify the evolution of the microsatellite instability signature between atypical hyperplasia/endometrial intraepithelial neoplasia and paired endometrial carcinomas, as well as to examine the relationship between mismatch repair immunohistochemistry, next-generation sequencing-based microsatellite instability detection, and polymerase chain reaction-based microsatellite instability detection in these specimens.

\section{Materials and methods}

\section{Case selection and histomorphologic evaluation}

This study was approved by the University of Chicago Institutional Review Board (\#17-173) with waiver of consent. Pathology reports were reviewed for all hysterectomies performed at the University of Chicago between January 2007 and July 2018. All hematoxylin and eosin-stained slides were reviewed for cases with (1) endometrioid histotype, mixed histotype with endometrioid elements (including dedifferentiated endometrial carcinoma), or undifferentiated histotype, (2) associated atypical hyperplasia/endometrial intraepithelial neoplasia component, and (3) known immunohistochemical mismatch repair deficiency in the carcinoma component. For inclusion in the study cohort, we required foci of both atypical hyperplasia/endometrial intraepithelial neoplasia and endometrial carcinoma that were sufficiently discrete for separate macro-dissection. For purposes of this study, we adopted the approach of Russo et al. [3], and considered lesions to constitute atypical hyperplasia/endometrial intraepithelial neoplasia if they met criteria for either endometrial intraepithelial neoplasia or atypical hyperplasia on review by two surgical pathologists (DBC and RRL). Hematoxylin and eosin-stained photomicrographs of each dissected specimen are available in Supplemental Fig. 1.

\section{UCM-OncoPlus next-generation sequencing}

Genomic DNA was isolated from macro-dissected formalin-fixed paraffin-embedded sections for the carcinoma and atypical hyperplasia/endometrial intraepithelial neoplasia components using the QIAamp DNA FFPE Tissue Kit (Qiagen, Valencia, CA) according to the manufacturer's instructions. Next-generation sequencing was performed using the targeted, hybrid capture 1,213-gene UCM-OncoPlus panel, as previously described [34]. In brief, following extraction, DNA was quantified using the Qubit fluorometric assay (Thermo Fisher Scientific, Foster City, CA) and further assessed for quantity and quality using a quantitative polymerase chain reaction assay (hgDNA Quantitation and QC kit, Kapa Biosystems, Wilmington, MA). Library preparation and sequencing were performed as previously described [34]. In brief, $100 \mathrm{ng}$ DNA was fragmented using the Covaris S2 (Covaris, Woburn, MA). The fragmented DNA was amplified using the KAPA HTP Library Preparation Kit (Kapa Biosystems) along with a set of patient-specific indexes (Roche, Indianapolis, IN). The pooled library was captured using a custom SeqCap EZ capture panel (Roche) featuring a collection xGen Lockdown Probes (IDT, Coralville, IA) for 1213 genes. The pooled, captured library was sequenced on the Illumina HiSeq 2500 system (Illumina, San Diego, CA) in rapid run mode $(2 \times 101 \mathrm{bp}$ paired end sequencing). Somatic mutation calling was performed across all 1213 genes using a custom in-house bioinformatics pipeline previously described [34]. Variants were annotated using Alamut Batch, Version 1.4 (Rouen, France).

\section{Next-generation sequencing-based microsatellite instability testing}

As part of UCM-OncoPlus, a microsatellite instability detection module using data from 336 incidentally captured homopolymers across the 1213 captured genes was utilized. A metric similar to that proposed by Kautto et al. was employed to quantify the stability of each homopolymer locus [24]. For each locus, distribution over different homopolymer lengths (normalized to a fraction of total depth at the locus) was generated. Then, the absolute value of the stepwise difference between that sample distribution and a normal distribution was calculated as a distance score $(d)$. The baseline distribution for normal samples was generated using average values across 23 non-tumor samples from our institutional population. To call a particular locus unstable, the ' $d$ ' score must be greater than the mean plus 3 standard deviations from the non-tumor population baseline. After excluding loci not meeting the quality depth threshold (depth of 50 reads with a Q30), the number of unstable loci in each specimen was expressed as a percentage. Thresholds for assigning microsatellite instability were defined using training sets of mismatch repair-retained 
and mismatch repair-deficient tumors, as determined by or immunohistochemistry. Samples with $<9 \%$ unstable loci were classified as microsatellite-stable, 9-15\% unstable loci as indeterminate, and $>15 \%$ unstable loci as microsatellite-unstable. Note that, unlike in polymerase chain reaction-based microsatellite instability calling, our next-generation sequencing-based method does not subclassify microsatellite-unstable tumors as microsatellite instability-low or microsatellite instability-high.

\section{Tumor mutational burden calculation}

Tumor mutational burden was calculated from OncoPlus data and quantified as mutations/megabase over a 1132 gene territory. Both synonymous and non-synonymous coding (exonic) variants were included. Variants that met any of the following criteria were excluded from the calculation: $<10 \%$ variant allele frequency, variants present in 1000 Genomes database (http://www.internationalgenome.org), and variants present in the Exome Aggregation Consortium (ExAC) database (http://exac.broadinstitute.org). Population database variants were rescued if there were $>10$ entries in the Catalogue of Somatic Mutations in Cancer (COSMIC) database (https://cancer.sanger.ac.uk/cosmic) with an ExAC frequency of $<0.001$ or if the variant was an insertion/ deletion and present in the ExAC database with QC flags (RF, AC0) and had an ExAC frequency no greater than 0.01 .

\section{Polymerase chain reaction-based microsatellite instability testing}

A fluorescent polymerase chain reaction-based assay, MSI Analysis System, (Version 1.2, Promega, Madison, WI) was used to detect microsatellite instability in a subset of cases (Cases 2, 3, 4, 6, 7, 8, 11, and 12). This system includes fluorescently labeled primers for co-amplification of seven markers including five mononucleotide repeat markers (BAT-25, BAT-26, NR-21, NR-24, and MONO-27) and two pentanucleotide repeat markers (Penta $\mathrm{C}$ and Penta $\mathrm{D}$ ). Polymerase chain reaction-based microsatellite instability testing was performed on a subset of tumor and matched normal ovarian formalin-fixed paraffin-embedded tissues from the patient's hysterectomy specimens. Resulting electropherograms were interpreted by two molecular pathologists (LLR, JPS).

\section{Mismatch repair protein immunohistochemistry}

For each case, immunohistochemistry for MLH1, MSH2, MSH6, and PMS2 was performed on the carcinoma and atypical hyperplasia/endometrial intraepithelial neoplasia components, using the same block selected for nextgeneration sequencing and using a previously published immunohistochemistry protocol [17]. In brief, the following antibody clones were used (each at 1:50 dilution): MLH1 (Dako M3640, mouse monoclonal, clone ES05), MSH2 (Dako M3639, mouse monoclonal, clone FE11), MSH6 (Dako M3646, mouse monoclonal, clone EP49), and PMS2 (Dako M3647, mouse monoclonal, clone EP51). Mismatch repair deficiency was defined by one of the following patterns: complete loss of tumor nuclear staining for both MLH1 and PMS2, both MSH2 and MSH6, MSH6 only, or PMS2 only, in the presence of a positive internal control. Mismatch repair retention was defined by tumor nuclei staining for all four proteins. Discrete foci of subclonal mismatch repair protein loss (heterogeneous expression) were noted, if present.

\section{Analysis of somatic mutations}

All variants identified by UCM-OncoPlus were prefiltered to remove any variants at $>1 \%$ frequency in the ExAC database, all synonymous variants, and splicing variants occurring $>2$ bp from the exon. Remaining variants were individually reviewed by a molecular pathologist to assess variant pathogenicity. Variants were considered pathogenic if they met either of the following criteria: loss-of-function mutations in known tumor suppressor genes (e.g. canonical splice sites, frameshift, or nonsense) or variants that occurred at a known hotspot location within the gene. The final list of pathogenic mutations for atypical hyperplasia/endometrial intraepithelial neoplasia and carcinoma components were compared for each case. Presence of one or more shared pathogenic somatic mutations and/or presence of $>10 \%$ shared unstable microsatellite loci were considered to constitute evidence of clonality in paired atypical hyperplasia/endometrial intraepithelial neoplasia and carcinoma specimens.

\section{Statistical analyses}

All statistical analyses were performed in GraphPad Prism (GraphPad Software, San Diego, CA). Contingency analyses between non-continuous variables were performed using Fisher's exact and chi-squared tests. Comparisons of two sets of continuous variables were performed using the (non-parametric) Mann-Whitney $U$ test, and comparisons of multiple sets of continuous variables were performed using the (non-parametric) Kruskal-Wallis one-way analysis of variance followed by Tukey's test of multiple comparisons. Comparisons of two continuous variables were performed using linear regression. 


\section{Results}

\section{Cohort characteristics}

The study cohort included 17 women who had undergone hysterectomy with bilateral salpingo-oophorectomy for endometrial carcinoma (Supplemental Table 1). Median age at diagnosis was 65 years (range, 44-77 years). Sixteen cases were stage $1 \mathrm{~A}$, and one case was stage $1 \mathrm{~B}$ [35]. Median tumor size was $3.6 \mathrm{~cm}$ (range, $0.8-11.5 \mathrm{~cm}$ ). Six tumors were FIGO grade 1, eight were FIGO grade 2, and three were FIGO grade 3. One FIGO grade 3 tumor (Case 11) had a substantial discrete component of grade 1 pattern growth, which was sequenced separately. No tumor showed extrauterine spread or lymph node involvement. No patients received neoadjuvant therapy.

\section{Mismatch repair protein immunohistochemistry}

Seventeen of 17 endometrial carcinoma specimens were mismatch repair-deficient by immunohistochemistry (MLH1/PMS2 loss in 16 cases; MSH2/MSH6 loss in 1 case [Case 2]). No cases exhibited heterogeneous mismatch repair protein expression. By immunohistochemistry, paired carcinoma and atypical hyperplasia/ endometrial intraepithelial neoplasia specimens showed concordant mismatch deficiency in 12/17 (71\%) cases (Fig. 1), including in $12 / 15$ cases with evidence for clonality between atypical hyperplasia/endometrial intraepithelial neoplasia and carcinoma specimens. In 4 cases (Cases 5, 6, 9, and 12) the atypical hyperplasia/ endometrial intraepithelial neoplasia component retained all mismatch repair proteins. Immunohistochemistry could not be performed in one case due to inadequate material in the sequenced tissue block. MLH1 promoter hypermethylation data were available in 5 cases with MLH1/PMS2 deficiency; promoter hypermethylation was present in all tested cases.

\section{Polymerase chain reaction-based microsatellite instability testing}

Polymerase chain reaction-based microsatellite instability calling was performed on 8 paired atypical hyperplasia/ endometrial intraepithelial neoplasia and endometrial carcinoma specimens. All carcinoma specimens were microsatellite instability-high. Among atypical hyperplasia/endometrial intraepithelial neoplasia specimens, three were microsatellite-stable (Cases 3, 6, and 12), two were microsatellite instability-low ( 1 of 5 loci unstable; Cases 7 and 8), and three were microsatellite instability-high (5 of 5 loci unstable; Cases 2, 4, and 11) (see Figs. 4 and 6).
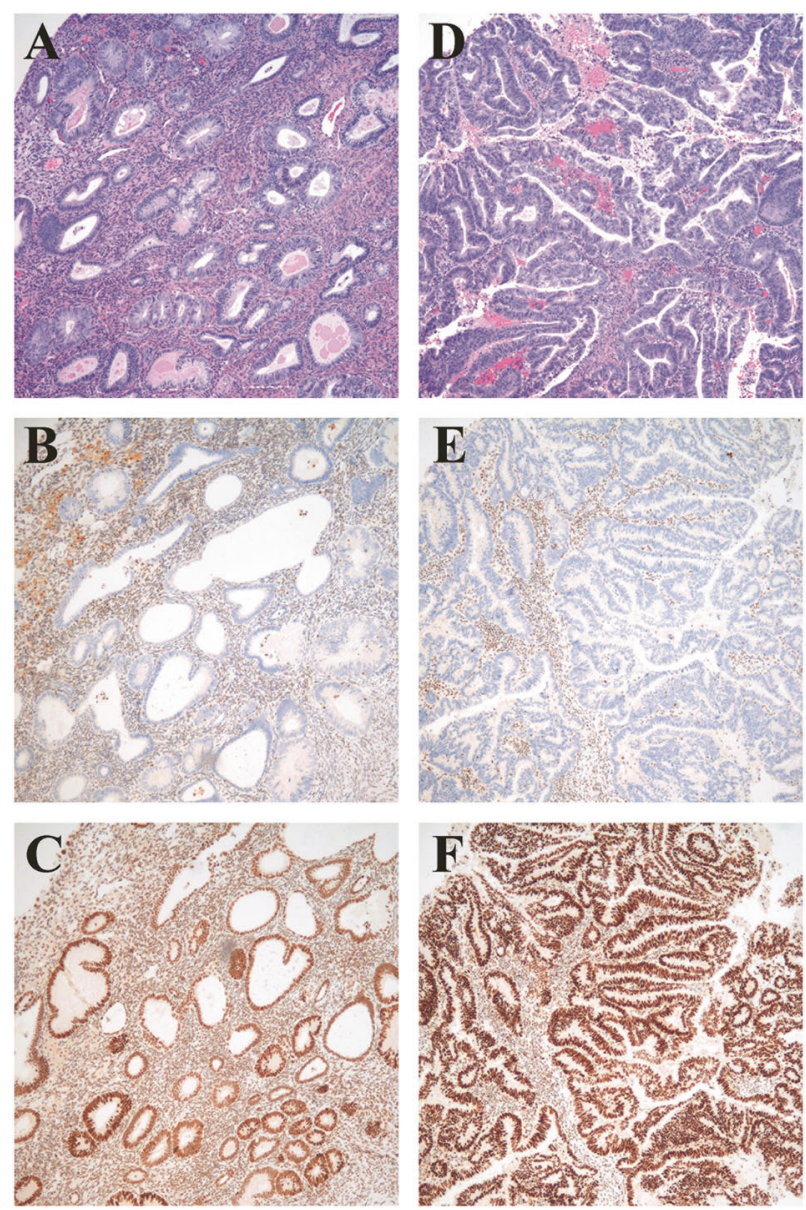

Fig. 1 Paired atypical hyperplasia/endometrial intraepithelial neoplasia and carcinoma show concordant mismatch repair protein expression profiles in Case 7. The atypical hyperplasia/endometrial intraepithelial neoplasia focus a shows complete loss of MLH1 (not shown) and PMS2 (b), with retention of MSH2 (not shown) and MSH6 (c). The carcinoma component (d) shows an identical pattern, with loss of MLH1 (not shown) and PMS2 (e) but retention of MSH2 (not shown) and MSH6 (f). (a, d: hematoxylin and eosin, original magnification $100 \times ; \mathbf{b}, \mathbf{c}, \mathbf{e}, \mathbf{f}:$ immunohistochemical stains with brown diaminobenzidine peroxidase-based detection, original magnification $\times 100$ )

\section{Next-generation sequencing-based microsatellite instability testing}

By next-generation sequencing-based microsatellite instability testing, 17/17 carcinoma specimens and 8/17 (47\%) atypical hyperplasia/endometrial intraepithelial neoplasia specimens exhibited microsatellite instability. Three out of $17(17.6 \%)$ atypical hyperplasia/endometrial intraepithelial neoplasia specimens were indeterminate, and 6/17 (35.3\%) were microsatellite-stable (Fig. 2a). In the single case with three separately sequenced elements, the percent unstable microsatellite loci increased sequentially from $47.3 \%$ in the atypical hyperplasia/endometrial intraepithelial neoplasia to $53.0 \%$ in the low-grade carcinoma and $64.3 \%$ in the high-grade carcinoma. The number of unstable 
A

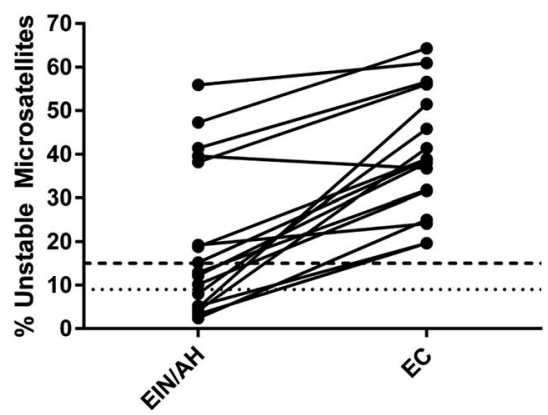

B

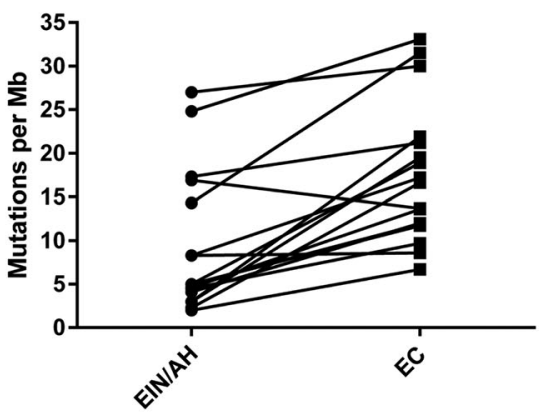

C

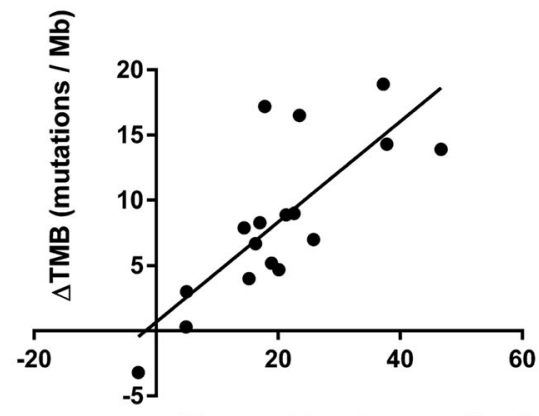

Fig. 2 a Endometrial carcinomas show significantly more unstable microsatellite loci than their paired atypical hyperplasia/endometrial intraepithelial neoplasia precursors $(p<0.0001$ by paired-samples $t$-test). All endometrial carcinoma specimens were microsatelliteunstable by next-generation sequencing-based calling (>15\% unstable loci, dashed line). Among atypical hyperplasia/endometrial intraepithelial neoplasia specimens, eight showed microsatellite instability, six were microsatellite-stable ( $<9 \%$ unstable loci, dotted line), and three were indeterminate ( $9-15 \%$ unstable loci). b Endometrial carcinomas also show significantly greater tumor mutational burden than paired atypical hyperplasia/endometrial intraepithelial neoplasia precursors ( $p<0.0001$ by paired-samples $t$-test). $\mathbf{c}$ The difference in tumor mutational burden between atypical hyperplasia/endometrial intraepithelial neoplasia and paired endometrial carcinoma specimens is significantly correlated with the difference in percentage of unstable microsatellites between atypical hyperplasia/endometrial intraepithelial neoplasia and paired carcinoma specimens $\left(p=0.0002 ; R^{2}=\right.$ 0.60). EIN/AH, atypical hyperplasia/endometrial intraepithelial neoplasia. EC, endometrial carcinoma. MB, megabase. TMB, tumor mutational burden. " $\Delta$ " indicates an arithmetic difference

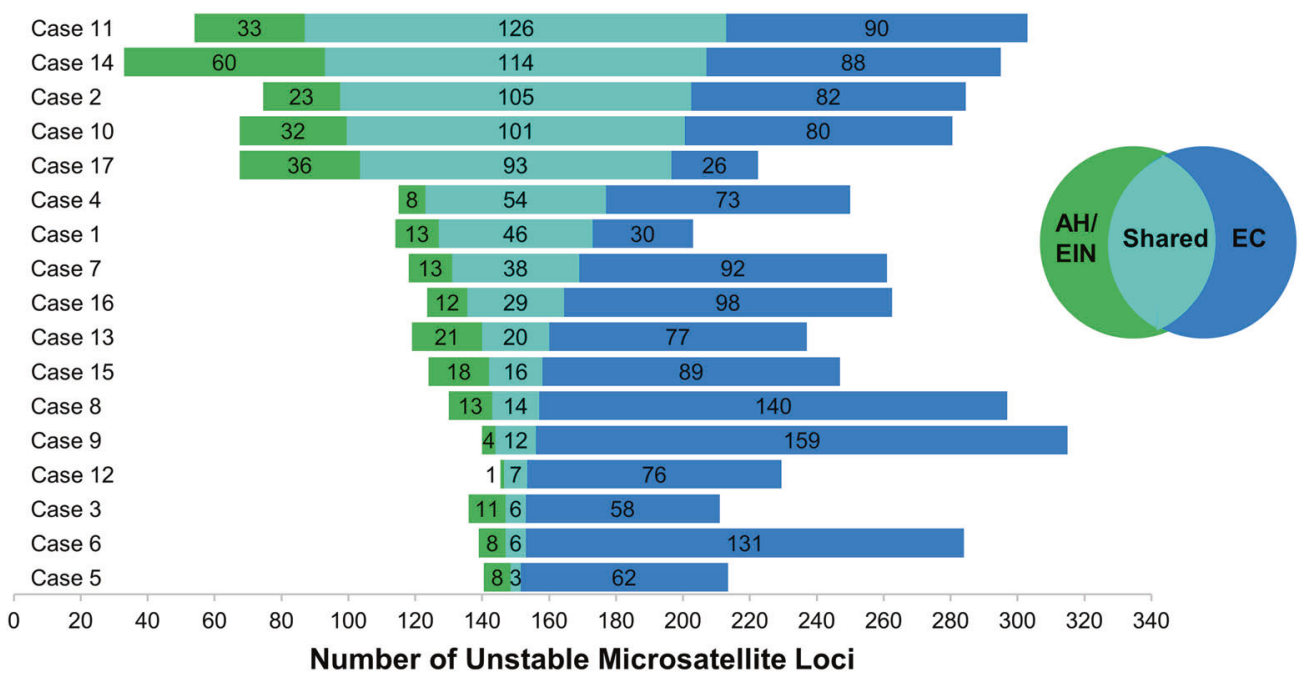

Fig. 3 Paired atypical hyperplasia/endometrial intraepithelial neoplasia and carcinoma specimens show substantial but variable overlap in percentage of unstable microsatellite loci, ranging from $4.1 \%$ shared unstable loci in Case 5 (3 shared unstable loci, of 73 total) to $51.7 \%$

microsatellite loci shared between paired atypical hyperplasia/endometrial intraepithelial neoplasia and carcinoma specimens ranged from 3 to 126 loci (mean, 46 unstable loci; median, 29 unstable loci) (Fig. 3). The percentage of unstable microsatellite loci shared between paired atypical hyperplasia/endometrial intraepithelial neoplasia and carcinoma specimens ranged from 4.1\% (Case 5: 3 shared unstable loci, of 73 total) to $51.7 \%$ (Case 1: 46 shared unstable loci, of 89 total). shared unstable loci in Case 1 (46 shared unstable loci, of 89 total). $\mathrm{AH} / \mathrm{EIN}$, atypical hyperplasia/endometrial intraepithelial neoplasia. $E C$, endometrial carcinoma

By next-generation sequencing-based microsatellite instability testing, the atypical hyperplasia/endometrial intraepithelial neoplasia elements showed a mean of $19.9 \%$ unstable microsatellite loci (median, $12.9 \%$ unstable microsatellite loci; range 2.4-55.9\%). The endometrial carcinoma specimens showed a mean of $40.0 \%$ unstable microsatellite loci (median, 38.7\% unstable microsatellite loci; range 19.6-64.3\%). Carcinoma specimens showed significantly more unstable microsatellite loci than paired 
atypical hyperplasia/endometrial intraepithelial neoplasia specimens (mean, 20.1 percentage points; median, 18.9 percentage points; $p<0.0001$; see Fig. 2a).

Only one case (Case 17) showed fewer unstable microsatellite loci in the carcinoma specimen than in the paired atypical hyperplasia/endometrial intraepithelial neoplasia specimen, with a decrement in unstable microsatellite loci of 2.8 percentage points. Case 17 was the only case in which the carcinoma and atypical hyperplasia/endometrial intraepithelial neoplasia specimens were dissected from different surgical pathology cases: the atypical hyperplasia/ endometrial intraepithelial neoplasia from a hysterectomy and the carcinoma from a biopsy performed 4 weeks prior, as no residual carcinoma was present in the hysterectomy.

\section{Concordance of Next-Generation Sequencing-based Microsatellite Instability Calling, Polymerase Chain Reaction-based Microsatellite Instability Calling, and Mismatch Repair Immunohistochemistry}

Polymerase chain reaction-based and next-generation sequencing-based microsatellite instability calling were concordant in all tested carcinoma specimens. In the atypical hyperplasia/endometrial intraepithelial neoplasia specimens, polymerase chain reaction-based and next-generation sequencing-based testing were concordant in 3 of 3 microsatellite instability-high specimens and in 3 of 3 microsatellite-stable specimens. Of the 2 atypical hyperplasia/endometrial intraepithelial neoplasia specimens called microsatellite instability-low by polymerase chain reactionbased testing, one was microsatellite-stable $(8 \%$ unstable loci) and one was microsatellite-unstable (15.2\% unstable loci) by next-generation sequencing (reference ranges: 0-9\% loci unstable $=$ microsatellite-stable; $9-15 \%=$ indeterminate; $>15 \%=$ microsatellite-unstable; Fig. 4).

Mismatch repair immunohistochemistry was concordant with both polymerase chain reaction-based and next-generation sequencing-based microsatellite instability testing in 17/17 carcinoma specimens. All 8 atypical hyperplasia/endometrial intraepithelial neoplasia specimens with microsatellite instability on next-generation sequencing-based testing were also mismatch repair-deficient by immunohistochemistry. Similarly, 3 out of 3 atypical hyperplasia/endometrial intraepithelial neoplasia specimens called indeterminate by next-generation sequencing-based microsatellite instability testing were mismatch repairdeficient by immunohistochemistry. In contrast, of 6 atypical hyperplasia/endometrial intraepithelial neoplasia specimens called microsatellite-stable by next-generation sequencing-based microsatellite instability testing, one was mismatch repair-deficient by immunohistochemistry (Case 8), four retained all mismatch repair proteins (Cases 5, 6, 9, and 12; Fig. 5), and one had insufficient tissue for
Case 2 Case 3 Case 4 Case 6 Case 7 Case 8 Case 11 Case 12

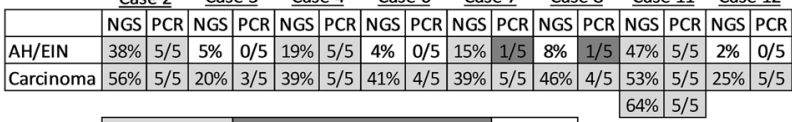

MSI-High

Fig. 4 Next-generation sequencing-based and polymerase chain reaction-based microsatellite instability calling methods are highly concordant in both endometrial carcinoma and atypical hyperplasia/ endometrial intraepithelial neoplasia specimens. In one discordant case (Case 7), an atypical hyperplasia/endometrial intraepithelial neoplasia specimen exhibited microsatellite instability by next-generation sequencing-based testing and was called microsatellite instabilitylow by polymerase chain reaction-based testing, and in a second discordant case (Case 8), an atypical hyperplasia/endometrial intraepithelial neoplasia specimen was called microsatellite-stable by nextgeneration sequencing-based testing and microsatellite instability-low by polymerase chain reaction-based testing. In both cases, this discrepancy reflects the borderline percentage of unstable microsatellites detected by the next-generation sequencing-based method, and both lesions appear to represent a transition between the microsatellitestable state and an outright microsatellite instability signature. Note that, in Case 11, the top "Carcinoma" cell represents the low-grade carcinoma component, and the bottom "Carcinoma" cell represents the high-grade carcinoma component. AH/EIN, atypical hyperplasia/ endometrial intraepithelial neoplasia. MSI, microsatellite instability. MSS, microsatellite-stable. NGS, Next-generation sequencing-based microsatellite instability calling method. PCR, Polymerase chain reaction-based microsatellite instability calling method
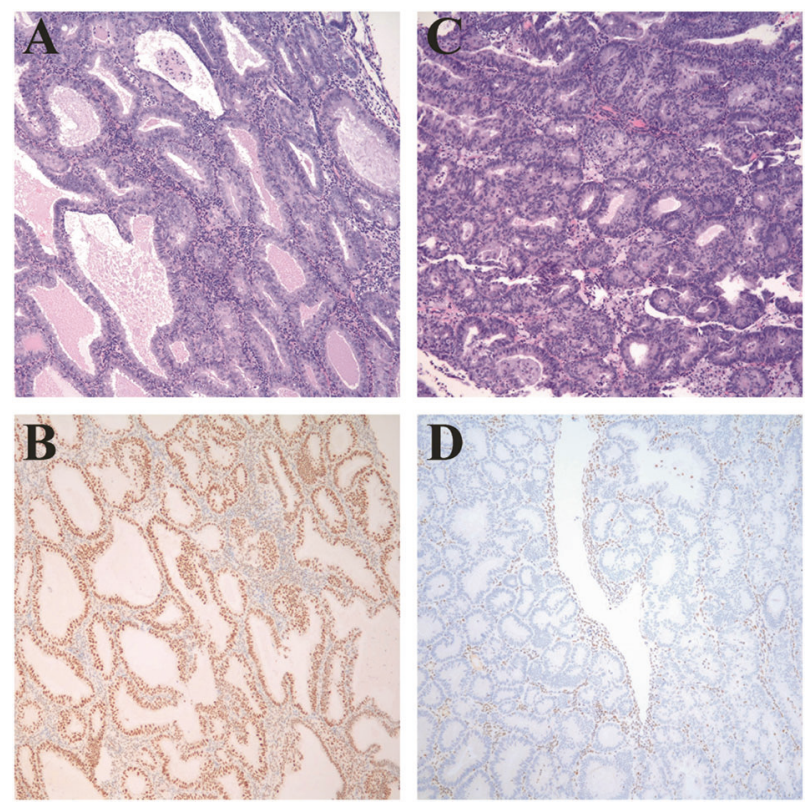

Fig. 5 Clonally related atypical hyperplasia/endometrial intraepithelial neoplasia and carcinoma pairs showed discordant mismatch repair protein expression profiles in few cases. In Case 5, the atypical hyperplasia/endometrial intraepithelial neoplasia focus (a) shows retention of PMS2 (b) as well as MLH1, MSH2, and MSH6 (not shown). The paired carcinoma (c) shows loss of MLH1 (not shown) and PMS2 (d) but retained expression of MSH2 and MSH6 (not shown). (a, c: hematoxylin and eosin, original magnification $\times 100$; b, d immunohistochemical stains with brown diaminobenzidine peroxidase-based detection, original magnification $\times 100$ ) 
immunohistochemistry (Case 3). Interestingly, among all microsatellite-stable atypical hyperplasia/endometrial intraepithelial neoplasia specimens, Case 8 showed the highest percentage $(8 \%)$ of unstable microsatellite loci and was called microsatellite instability-low by polymerase chain reaction-based testing (1/5 loci unstable).

\section{Correlation of microsatellite instability with clinicopathologic features}

Percentage of unstable loci in carcinoma specimens was significantly related to tumor grade, with FIGO grade 1 carcinoma showing significantly fewer unstable microsatellite loci than FIGO 2 or FIGO 3 carcinoma $(p=0.017)$. In contrast, percentage of unstable loci in atypical hyperplasia/endometrial intraepithelial neoplasia specimens was not significantly related to (1) grade of the paired carcinoma ( $p=0.49$; note that two grade 3 carcinomas were associated with microsatellite-stable atypical hyperplasia/endometrial intraepithelial neoplasia), (2) whether the atypical hyperplasia/endometrial intraepithelial neoplasia had been dissected from the same or different block as the paired carcinoma $(p=0.99)$, or (3) tumor size $(p=0.54)$. Age at diagnosis was not significantly related to the percentage of unstable loci in the atypical hyperplasia/endometrial intraepithelial neoplasia or carcinoma components $(p=0.37$ and $=0.57$, respectively). In an unsupervised clustering analysis, the sole case with MSH2/MSH6 deficiency (Case
2) did not show a distinct pattern of unstable microsatellite loci, compared to the 16 MLH1/PMS2-deficient cases (data not shown).

\section{Recurrent variants in atypical hyperplasia/ endometrial intraepithelial neoplasia and endometrioid endometrial carcinoma}

Among recurrently mutated genes in endometrioid endometrial carcinoma [36-38], 42 total pathogenic somatic mutations were identified in the 17 atypical hyperplasia/ endometrial intraepithelial neoplasia specimens and 83 total pathogenic somatic mutations in 17 endometrial carcinoma specimens, with at least one shared mutation in 13/17 (76.5\%) paired atypical hyperplasia/endometrial intraepithelial neoplasia and carcinoma specimens (Fig. 6). PTEN was the most commonly mutated gene, with 22 pathogenic mutations, detected in 17 out of 17 carcinoma specimens and 11 out of 17 atypical hyperplasia/endometrial intraepithelial neoplasia specimens. At least 1 PTEN mutation was shared by paired atypical hyperplasia/endometrial intraepithelial neoplasia and carcinoma specimens in 10 of 17 cases. At least one PTEN frameshift mutation occurred in 9 cases, and included two recurrent frameshifts (p.Lys267Argfs $* 9$ in five cases, and p.Asn323Metfs $* 21$ in two cases) and four frameshifts occurring in only one case (p.Glu235Alafs*7, p.Asn262Glnfs*35, p.Ile101Argfs*8, and p.Tyr225Phefs*17).

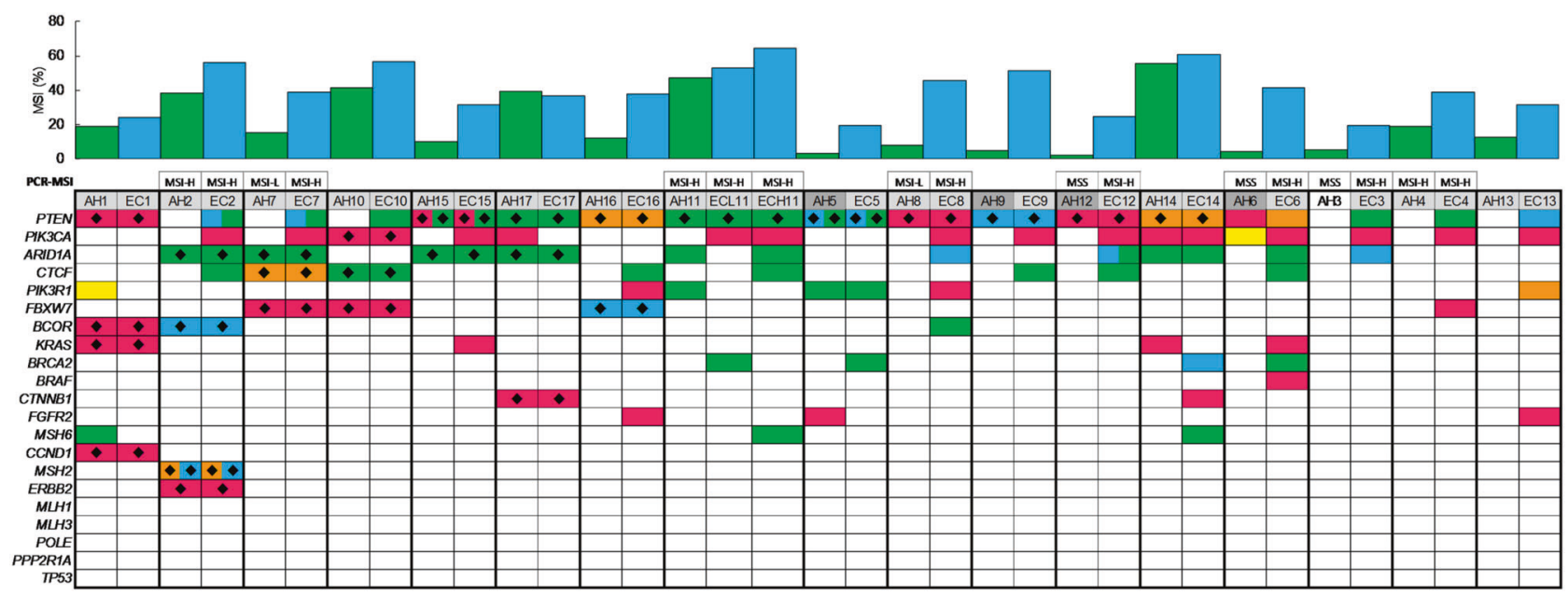

MMRP-deficient MMRP-retained missense nonsense frameshift in-frame splicing $\bullet=$ shared between AH\& EC

Fig. 6 Comparison of somatic mutations and percentage of unstable microsatellite loci in paired atypical hyperplasia/endometrial intraepithelial neoplasia and carcinoma specimens. Thirteen of 17 paired atypical hyperplasia/endometrial intraepithelial neoplasia and carcinoma specimens shared at least one pathogenic somatic mutation in a gene known to be significant in pathogenesis of endometrioid endometrial carcinoma [36-38]. A single case (Case 2) showed shared biallelic pathogenic mutations in a mismatch repair protein gene (MSH2), which was not present on germline testing. In two cases (Cases 4 and 13) lacking a shared somatic mutation in an endometrial carcinoma-related gene, substantial overlap of unstable microsatellite loci was observed between atypical hyperplasia/endometrial intraepithelial neoplasia and carcinoma specimens, favoring a clonal relationship. $\mathrm{AH}$, atypical hyperplasia/endometrial intraepithelial neoplasia. EC, endometrial carcinoma. NGS-MSI (\%), percentage of unstable microsatellite loci on next-generation sequencing-based microsatellite instability testing. PCR-MSI, polymerase chain reactionbased microsatellite testing result (MSS, microsatellite stable; MSI-L, microsatellite instability-low; MSI-H, microsatellite instability-high) 
PIK3CA was the second-most commonly mutated gene, with 17 pathogenic mutations detected in 13 out of 17 carcinoma specimens and 4 out of 17 atypical hyperplasia/ endometrial intraepithelial neoplasia specimens, with a shared PIK3CA mutation identified in just one atypical hyperplasia/endometrial intraepithelial neoplasia and carcinoma pair. ARIDIA was the third-most commonly mutated gene, with 13 pathogenic mutations in 10 out of 17 carcinoma specimens and 6 out of 17 atypical hyperplasia/ endometrial intraepithelial neoplasia specimens, including shared mutations in 4 atypical hyperplasia/endometrial intraepithelial neoplasia and carcinoma pairs. Somatic pathogenic mutations shared by paired atypical hyperplasia/ endometrial intraepithelial neoplasia and carcinoma specimens were also observed in CTCF, FBXW7, BCOR, KRAS, $C T N N B 1, C C N D 1$, and ERBB2. In one case (Case 2) with MSH2/MSH6 loss, genetic testing for germline mutations in $M S H 2, M S H 6$, and EPCAM was negative. Double somatic mutations in $\mathrm{MSH} 2$ were detected in both the atypical hyperplasia/endometrial intraepithelial neoplasia and carcinoma specimens.

Among those genes recurrently mutated in endometrioid endometrial carcinoma [36-38] (see Fig. 6), our carcinoma specimens contained significantly more pathogenic somatic mutations than their paired atypical hyperplasia/endometrial intraepithelial neoplasia specimens (median: 5 mutations vs 3 mutations, respectively; $p=0.0001)$. Furthermore, the number of pathogenic mutations in these genes of interest was significantly correlated with the percentage of unstable microsatellite loci in a given atypical hyperplasia/ endometrial intraepithelial neoplasia or carcinoma specimen $(p=0.039)$. The number of pathogenic mutations in these genes of interest was not significantly different in FIGO grade 1, grade 2, and grade 3 carcinomas.

\section{Tumor mutational burden}

The atypical hyperplasia/endometrial intraepithelial neoplasia elements showed a mean of 9.1 mutations/megabase (median, 5.0 mutations/megabase; range 2.0-27.0 mutations/megabase). The endometrial carcinoma specimens showed a mean of 17.5 mutations/megabase (median, 16.6 mutations/megabase; range 6.7-33.1 mutations/megabase). Carcinoma specimens showed a significantly higher tumor mutational burden than paired atypical hyperplasia/endometrial intraepithelial neoplasia specimens $(p<0.0001$; Fig. 2b). Tumor mutational burden was significantly correlated with the percentage of unstable microsatellite loci in atypical hyperplasia/endometrial intraepithelial neoplasia specimens $\left(p<0.0001, R^{2}=0.87\right)$ and in carcinoma specimens $\left(p<0.0001, R^{2}=0.67\right)$. The increment in the percentage of unstable microsatellite loci between atypical hyperplasia/endometrial intraepithelial neoplasia and carcinoma specimens in a given case and the increment in tumor mutational burden in that case were significantly correlated ( $p=0.0002, R^{2}=0.60 ;$ Fig. $2 \mathrm{c}$ ).

\section{Discussion}

This multimodal investigation of paired atypical hyperplasia/endometrial intraepithelial neoplasia and endometrioid endometrial carcinoma specimens provides novel insights into the role of mismatch repair deficiency in the oncogenesis of a subset of endometrial carcinomas, and further clarifies the relationship between immunohistochemical testing for mismatch repair proteins and molecular testing for microsatellite instability.

Data first published more than 20 years ago [15, 16, 39] support a model of endometrial carcinogenesis wherein a subset of tumors undergoes early hypermethylation of the MLH1 gene, followed by expression of the microsatellite instability signature. Our study provides additional insights. First, our data indicate that, in clonally related atypical hyperplasia/endometrial intraepithelial neoplasia and endometrial carcinomas, loss of mismatch repair protein expression is an early event, already present in the atypical hyperplasia/endometrial intraepithelial neoplasia precursor in 12 out of $15(80 \%)$ cases with evidence for clonality. Even in mismatch repair-deficient endometrioid endometrial carcinoma, however, loss of mismatch repair does not appear to be the initiating oncogenic event, as shown by 3 cases with a mismatch repair-proficient, microsatellitestable atypical hyperplasia/endometrial intraepithelial neoplasia precursor and a clonally related mismatch repairdeficient, microsatellite-unstable carcinoma. Additional important early molecular events in endometrial carcinogenesis are further suggested by shared PTEN mutations in atypical hyperplasia/endometrial intraepithelial neoplasia and carcinoma specimens in 13 out of 15 cases with evidence of clonality - a finding in keeping with recently published work [3]. Conversely, although PIK3CA mutations were present in 13 out of 17 carcinomas, they were present in only 4 atypical hyperplasia/endometrial intraepithelial neoplasia specimens and were shared between paired atypical hyperplasia/endometrial intraepithelial neoplasia and carcinoma specimens in just one case, suggesting that mutations in $P I K 3 C A$ are a relatively late event in endometrial carcinogenesis.

Importantly, our data further indicate that the microsatellite instability molecular signature develops in mismatch repair-deficient tumors when sufficient time permits accumulation of variant microsatellite lengths, over multiple cycles of cell replication. Among 12 mismatch repairdeficient atypical hyperplasia/endometrial intraepithelial neoplasia specimens, 3 were microsatellite-stable, 3 
indeterminate, and 6 microsatellite-unstable by nextgeneration sequencing-based testing. These cases might be considered, respectively, as "early," "middle," and "late" snapshots of the molecular sequelae of loss of mismatch repair function. In contrast to the variable relationship between loss of mismatch repair proteins and microsatellite instability signature in atypical hyperplasia/endometrial intraepithelial neoplasia specimens, mismatch repair loss and microsatellite instability signature are much more strongly correlated in carcinoma specimens, with concordance in all 17 carcinomas in this study. This strong correlation suggests that the accumulation of unstable microsatellites is a sine qua non for progression from atypical hyperplasia/endometrial intraepithelial neoplasia to frank carcinoma in mismatch repair-deficient cases. Together, these data offer further support for a complex phylogenetic relationship between atypical hyperplasia/ endometrial intraepithelial neoplasia and associated endometrioid endometrial carcinoma, in which carcinoma arises from a subclone within the atypical hyperplasia/endometrial intraepithelial neoplasia, with both the emergent carcinoma and the originating atypical hyperplasia/endometrial intraepithelial neoplasia then continuing to evolve and accumulate additional non-shared mutations [3].

Multiple older molecular studies of paired clonal atypical hyperplasia/endometrial intraepithelial neoplasia and carcinoma specimens are available [4-6, 15, 16, 39], but we are aware of only one more recent study using next-generation sequencing to examining clonal evolution of atypical hyperplasia/endometrial intraepithelial neoplasia and carcinoma [3]. In a small but well-designed study, Russo and colleagues sequenced six cases of paired atypical hyperplasia/endometrial intraepithelial neoplasia and endometrial carcinoma (including three mismatch repair-deficient tumors) and showed clonality in all cases on the basis of shared somatic mutations, shared copy number alterations, and concordant mismatch repair protein expression between the atypical hyperplasia/endometrial intraepithelial neoplasia and carcinoma components. They did not utilize next-generation sequencing-based microsatellite instability calling. In keeping with our data, their report finds that loss of mismatch repair function is an early event in mismatch repair-deficient endometrial cancers. However, our substantially larger cohort provides further insight. Namely, approximately one fifth of mismatch repair-deficient endometrioid endometrial carcinomas are clonally related to atypical hyperplasia/endometrial intraepithelial neoplasia with retained expression of mismatch repair proteins, indicating that mismatch repair loss is not a truncal molecular event in all cases, and suggesting that "mismatch repair-deficient" endometrial carcinoma group described by the Cancer Genome Atlas group harbors molecular heterogeneity, although the clinical significance of this variability remains unclear.
Our study further clarifies the nature of the microsatellite instability-low phenotype in polymerase chain reaction-based microsatellite instability assay. The two cases called microsatellite instability-low by polymerase chain reaction-based testing in our study were called either microsatellite-stable (Case 8 , with $8 \%$ unstable loci) or microsatellite-untable (Case 7, with $15.2 \%$ unstable loci) by next-generation sequencing-based microsatellite instability testing. These data suggest that the microsatellite instability-low phenotype may not be a distinct molecular subgroup, but instead may represent mismatch repair-deficient tumors in transition to an outright microsatellite-unstable molecular phenotype.

Our data support that somatic mutations in mismatch repair protein genes are rare in the setting of MLH1/PMS2deficient endometrial carcinomas. Among 16 MLH1/PMS2deficient cases, no somatic mutations were seen in $M L H 1$ or $P M S 2$, consistent with $M L H 1$ promoter hypermethylation in these cases. In contrast, the single MSH2/MSH6-deficient case in our cohort had biallelic somatic $M S H 2$ mutations, emphasizing the importance of tumor sequencing in mismatch repair-deficient tumors with no detectable germline mutation. Three MLH1/PMS2-deficient cases (Cases 1, 11, and 14) showed a heterozygous frameshift mutation in MSH6 in either the atypical hyperplasia/endometrial intraepithelial neoplasia or carcinoma specimen, arising in a polyC homopolymer at codon 1088 of $\mathrm{MSH6}$ as a passenger mutation secondary to instability of coding microsatellites [40]. Note that unstable coding microsatellites can also introduce frameshifts in PTEN [40], as we also observed. Specifically, we identified two PTEN frameshifts occurring in multiple cases and in association with poly-A homopolymers, as well as four PTEN frameshifts unique to individual cases and not associated with homopolymers. This indicates that some PTEN frameshift mutations in mismatch repair-deficient endometrial carcinomas are secondary to microsatellite instability, whereas some PTEN frameshifts occur independent of microsatellite instability, in keeping with the Cancer Genome Atlas study of endometrial cancers, in which $30 \%$ of PTEN mutations in microsatellite-stable carcinomas were frameshift mutations [36].

Our data also show a significant increase in overall tumor mutational burden between paired atypical hyperplasia/ endometrial intraepithelial neoplasia and carcinoma, analogous to the increase in unstable microsatellite loci between paired specimens. Furthermore, the increment in the percentage of unstable microsatellites between atypical hyperplasia/endometrial intraepithelial neoplasia and carcinoma specimens in a given case correlated strongly with the increment in overall tumor mutation burden in that case. Although this result is not unexpected, given the role that mismatch repair deficiency plays in the accumulation of tumor mutations, these data nonetheless provide additional evidence for accumulation of mutations during endometrial 
carcinogenesis. Practically, this result also highlights the importance of carefully selecting tumor tissue for sequencing, particularly as the clinical applications of tumor mutational burden are further clarified [33]. This caveat is especially relevant, as tumor mutational burden (unlike mismatch repair deficiency) has no immunohistochemical cognate for correlation with molecular results [41, 42].

In addition to their implications for endometrial carcinogenesis, these data are also clinically relevant. First, they support earlier observations $[15,16]$ that abnormal mismatch repair immunohistochemistry or microsatellite instability results in an atypical hyperplasia/endometrial intraepithelial neoplasia specimen correlate strongly with an abnormal result in the paired carcinoma, which may justify mismatch repair immunohistochemistry or microsatellite instability testing in atypical hyperplasia/endometrial intraepithelial neoplasia for diagnostic or prognostic purposes [7,9], particularly in cases where carcinoma tissue is not available for testing.

Next-generation sequencing-based methods for microsatellite instability detection are of substantial clinical value. As noted above, the ability to evaluate hundreds of microsatellite loci with a single test significantly reduces the tumor-specific bias inherent to small panels of microsatellite loci [28]. Tellingly, Hause and colleagues, in their analysis of the microsatellite fingerprints of 18 different tumor types, found only $30 \%$ overlap in unstable microsatellite loci between endometrial and colorectal carcinoma [25]. This is in keeping with a recent report that, even when using nextgeneration sequencing-based methods, accuracy of Lynch syndrome screening is improved when different cutoffs are used for calling MSI in endometrial versus colorectal carcinoma [32]. Despite the complex landscape of microsatellite instability across tumor types, large-panel next-generation sequencing-based microsatellite instability testing has greater adaptability than smaller polymerase chain reaction-based microsatellite instability panels; provides more granular locus-specific data, which may prove to have further diagnostic, prognostic, or predictive value; and opens the door to improved single-test molecular classification of endometrial carcinomas, as promulgated in the ProMisE model $[12,13]$.

Our study has limitations to consider. First, assessments of clonality are more complicated by the absence of matched germline sequencing for each tumor. However, we strictly filtered potential germline variants, and identified shared somatic mutations in 13/17 paired atypical hyperplasia/ endometrial intraepithelial neoplasia and carcinoma specimens, providing evidence for clonality. In two additional cases, paired atypical hyperplasia/endometrial intraepithelial neoplasia and carcinomas shared a significant proportion of unstable microsatellite loci (17\% in Case 13 and $40 \%$ in Case 4), despite lacking shared somatic mutations.
Second, examining each case (with the exception of Case 17) at a single point in time precludes conclusions about the rate at which unstable microsatellites accumulate, the timeframe for progression from loss of mismatch repair protein expression to a true microsatellite instability signature, and the factors affecting the kinetics of unstable microsatellite accumulation. However, our data show that spatial proximity of the atypical hyperplasia/endometrial intraepithelial neoplasia and paired endometrial carcinoma does not predict the microsatellite instability status in the atypical hyperplasia/ endometrial intraepithelial neoplasia. Additional studies of patients with multiple diagnoses of atypical hyperplasia/ endometrial intraepithelial neoplasia in sequential endometrial biopsies would be valuable in further clarifying the kinetics of unstable microsatellite accumulation.

Third, our cohort had significantly higher representation of MLH1/PMS2-deficient tumors, compared to previous studies with age-restricted cohorts, which are substantially more enriched in Lynch syndrome patients and in patients with mismatch repair protein deficiencies other than MLH1/ PMS2 [7, 9]. The preponderance of MLH1/PMS2-deficient cases in our cohort limits extrapolation to cases with other mismatch repair protein deficiencies and to Lynch syndrome patients, which may show some biological differences. However, because MLH1-hypermethylated MLH1/ PMS2-deficient tumors account for the majority of mismatch repair-deficient endometrial carcinomas [43], our data have the potential to guide further investigation and management of a significant proportion of all endometrial carcinomas. Similarly, our cohort included only endometrioid-type endometrial carcinomas. While endometrioid tumors constitute up to $93 \%$ of mismatch repairdeficient endometrial carcinomas, mismatch repair deficiency and microsatellite instability are also observed in other types of endometrial carcinoma [12, 13], and further study is needed to generalize our findings to all mismatchdeficient endometrial carcinomas and their precursors.

Finally, because we used mismatch repair deficiency in the carcinoma as a criterion for inclusion, we are unable to comment on the potential for microsatellite-stable endometrial carcinomas to develop from microsatellite-unstable atypical hyperplasia/endometrial intraepithelial neoplasia precursors, although existing data argue that if this occurs, it is exceptionally rare $[3,7,15,16]$ and would likely result from spatial heterogeneity of the sequenced tumor. Analogous studies examining the relationship between paired atypical hyperplasia/endometrial intraepithelial neoplasia and carcinoma specimens in other molecular subtypes of endometrial carcinoma would be of interest.

This next-generation sequencing-based study of paired atypical hyperplasia/endometrial intraepithelial neoplasia and carcinoma specimens shows progressive accumulation of unstable microsatellite loci following loss of mismatch 
repair protein expression, in this subset of endometrial carcinomas. Next-generation sequencing-based testing offers new opportunities to better understand the development of endometrial carcinomas, with implications for tumor surveillance, diagnosis, and management. Additional studies are needed to clarify the relationship between carcinomas and their precursors in other molecular subtypes of endometrial carcinoma, and to examine serial atypical hyperplasia/endometrial intraepithelial neoplasia and carcinoma specimens from conservatively managed patients to better clarify the time course of molecular alterations in endometrial carcinogenesis.

\section{Compliance with ethical standards}

Conflict of interest The authors declare that they have no conflict of interest.

Publisher's note: Springer Nature remains neutral with regard to jurisdictional claims in published maps and institutional affiliations.

\section{References}

1. Siegel RL, Miller KD, Jemal A. Cancer statistics, 2019. CA Cancer J Clin. 2019;69:7-34.

2. Felix AS, Yang HP, Bell DW, Sherman ME. Epidemiology of endometrial carcinoma: etiologic importance of hormonal and metabolic influences. Adv Exp Med Biol. 2017;943:3-46.

3. Russo M, Broach J, Sheldon K, Houser KR, Liu DJ, Kesterson J, et al. Clonal evolution in paired endometrial intraepithelial neoplasia/atypical hyperplasia and endometrioid adenocarcinoma. Hum Pathol. 2017;67:69-77.

4. Werner HM, Berg A, Wik E, Birkeland E, Krakstad C, Kusonmano $\mathrm{K}$, et al. ARID1A loss is prevalent in endometrial hyperplasia with atypia and low-grade endometrioid carcinomas. Mod Pathol. 2013;26:428-34.

5. Baloglu H, Cannizzaro LA, Jones J, Koss LG. Atypical endometrial hyperplasia shares genomic abnormalities with endometrioid carcinoma by comparative genomic hybridization. Hum Pathol. 2001;32:615-22.

6. Muslumanoglu HM, Oner U, Ozalp S, Acikalan MF, Yalcin OT, Ozdemir M, et al. Genetic imbalances in endometrial hyperplasia and endometrioid carcinoma detected by comparative genomic hybridization. Eur J Obstet Gynecol Reprod Biol. 2005;120:107-14.

7. Sutter C, Dallenbach-Hellweg G, Schmidt D, Baehring J, Bielau S, von Knebel Doeberitz M, et al. Molecular analysis of endometrial hyperplasia in HNPCC-suspicious patients may predict progression to endometrial carcinoma. Int $\mathrm{J}$ Gynecol Pathol. 2004;23:18-25.

8. Byun JM, Jeong DH, Kim YN, Cho EB, Cha JE, Sung MS, et al. Endometrial cancer arising from atypical complex hyperplasia: the significance in an endometrial biopsy and a diagnostic challenge. Obstet Gynecol Sci. 2015;58:468-74.

9. Lucas E, Chen H, Molberg K, Castrillon DH, Rivera Colon G, Li $\mathrm{L}$, et al. Mismatch repair protein expression in endometrioid intraepithelial neoplasia/atypical hyperplasia: should we screen for lynch syndrome in precancerous lesions? Int J Gynecol Pathol. 2018. https://doi.org/10.1097/PGP.0000000000000557.

10. van der Putten LJM, van Hoof R, Tops BBJ, Snijders MPLM, van der Berg-van Erp SH, van der Wurff AAM. et al. Molecular profiles of benign and (pre)malignant endometrial lesions. Carcinogenesis. 2017;38:329-35.

11. Zaino R, Carinelli SG, Ellenson LH, Eng C, Katabuchi H, Konishi I, et al. Epithelial tumours and precursors. In: Kurman RJ, et al., editors. WHO classification of tumours of the female reproductive organs. 4th edn. Lyon, France: IARC; 2014. p. 125-6.

12. Talhouk A, McConechy MK, Leung S, Yang W, Lum A, Senz J, et al. Confirmation of ProMisE: a simple, genomics-based clinical classifier for endometrial cancer. Cancer. 2017;123: 802-13.

13. Kommoss S, McConechy MK, Kommoss F, Leung S, Bunz A, Magrill J, et al. Final validation of the ProMisE molecular classifier for endometrial carcinoma in a large population-based case series. Ann Oncol. 2018;29:1180-8.

14. MacDonald ND, Salvesen HB, Ryan A, Iversen OE, Aklen LA, Jacobs IJ. Frequency and prognostic impact of microsatellite instability in a large population-based study of endometrial carcinomas. Cancer Res. 2000;60:1750-2.

15. Esteller M, Catasus L, Matias-Guiu X, Mutter GL, Prat J, Baylin $\mathrm{SB}$, et al. hMLH1 promoter hypermethylation is an early event in human endometrial tumorigenesis. Am J Pathol. 1999; 155:1767-72.

16. Levine RL, Cargile CB, Blazes MS, van Rees B, Kurman RJ, Ellenson LH. PTEN mutations and microsatellite instability in complex atypical hyperplasia, a precursor lesion to uterine endometrioid carcinoma. Cancer Res. 1998;58:3254-8.

17. Chapel DB, Yamada SD, Cowan M, Lastra RR. Immunohistochemistry for mismatch repair protein deficiency in endometrioid endometrial carcinoma yields equivalent results when performed on endometrial biopsy/curettage or hysterectomy specimens. Gynecol Oncol. 2018;149:570-4.

18. Vierkoetter KR, Kagami LA, Ahn HJ, Shimizu DM, Terada KY. Loss of mismatch repair protein expression in unselected endometrial adenocarcinoma precursor lesions. Int J Gynecol Cancer. 2016;26:228-32.

19. Bonadona V, Bonaiti B, Olschwang S, Grandjouan S, Huiart L, Longy $\mathrm{M}$, et al. Cancer risks associated with germline mutations in MLH1, MSH2, and MSH6 genes in Lynch syndrome. J Am Med Assoc. 2011;305:2304-10.

20. Barrow E, Robinson L, Alduaij W, Shenton A, Clancy T, Lalloo $\mathrm{F}$, et al. Cumulative lifetime incidence of extracolonic cancers in Lynch syndrome: a report of 121 families with proven mutations. Clin Genet. 2009;75:141-9.

21. Bartosch C, Clarke B, Bosse T. Gynaecological neoplasms in common familial syndromes (Lynch and HBOC). Pathology. 2018;50:222-37.

22. Wu X, Snir O, Rottmann D, Wong S, Buza N, Hui P. Minimal microsatellite shift in microsatellite instability high endometrial cancer: a significant pitfall in diagnostic interpretation. Mod Pathol. 2019;32:650-8.

23. McConechy MK, Talhouk A, Li-Chang HH, Leung S, Huntsman DG, Gilks CB, et al. Detection of DNA mismatch repair (MMR) deficiencies by immunohistochemistry can effectively diagnose the microsatellite instability (MSI) phenotype in endometrial carcinomas. Gynecol Oncol. 2015;137:306-10.

24. Stelloo E, Jansen AML, Osse EM, Nout RA, Creutzberg CL, Ruano D, et al. Practical guidance for mismatch repairdeficiency testing in endometrial cancer. Ann Oncol. 2017; 28:96-102.

25. Kautto EA, Bonneville R, Miya J, Yu L, Krook MA, Reeser JW, et al. Performance evaluation for rapid detection of pancancer microsatellite instability with MANTIS. Oncotarget. 2017;8:7452-63.

26. Hause RJ, Pritchard CC, Shendure J, Salipante SJ. Classification and characterization of microsatellite instability across 18 cancer types. Nat Med. 2016;22:1342-50. 
27. Salipante SJ, Scroggins SM, Hampel HL, Turner EH, Pritchard CC. Microsatellite instability detection by next generation sequencing. Clin Chem. 2014;60:1192-9.

28. Niu B, Ye K, Zhang Q, Lu C, Xie M, McClellan MD, et al. MSIsensor: microsatellite instability detection using paired tumornormal sequence data. Bioinformatics. 2014;30:1015-6.

29. Dong F, Costigan DC, Howitt BE. Targeted next-generation sequencing in the detection of mismatch repair deficiency in endometrial cancers. Mod Pathol. 2019;32:252-7.

30. Le DT, Durham JN, Smith KN, Wang H, Bartlett BR, Aulakh LK, et al. Mismatch repair deficiency predicts response of solid tumors to PD-1 blockade. Science. 2017;357:409-13.

31. Lee V, Murphy A, Le DT, Diaz LA Jr. Mismatch repair deficiency and response to immune checkpoint blockade. Oncologist. 2016;21:1200-11.

32. US, Administration FaD. Keytruda BLA 125514/S-14, Supplement -14. https://www.accessdata.fda.gov/drugsatfda_ docs/appletter/2017/125514Orig1s014ltr.pdf. 23 May 2017. Accessed: 19 February 2019.

33. Goodman AM, Kato S, Bazhenova L, Patel SP, Frampton GM, Miller V, et al. Tumor mutational burden as an independent predictor of response to immunotherapy in diverse cancers. Mol Cancer Ther. 2017;16:2598-608.

34. Kadri S, Long BC, Mujacic I, Zhen CJ, Wurst MN, Sharma S, et al. Clinical validation of a next-generation sequencing genomic oncology panel via cross-platform benchmarking against established amplicon sequencing assays. J Mol Diagn. 2017; 19:43-56.

35. Amin M, Edge S, Greene F, Byrd D. AJCC cancer staging manual. 8th ed. Springer: New York; 2017.
36. Kandoth C, Schultz N, Cherniack AD, Akbani R, Liu Y, Shen H, et al. Integrated genomic characterization of endometrial carcinoma. Nature. 2013;497:67-73.

37. Kandoth C, McLellan MD, Vandin F, Ye K, Niu B, Lu C, et al. Mutational landscape and significance across 12 major cancer types. Nature. 2013;502:333-9.

38. Lawrence MS, Stojanov P, Mermel CH, Robinson JT, Garraway LA, Golub TR, et al. Discovery and saturation analysis of cancer genes across 21 tumour types. Nature. 2014;505:495-501.

39. Mutter GL, Boynton KA, Faquin WC, Ruiz RE, Jovanovic AS. Allelotype mapping of unstable microsatellites establishes direct lineage continuity between endometrial precancers and cancer. Cancer Res. 1996;56:4483-6.

40. Woerner SM, Gebert J, Yuan YP, Sutter C, Ridder R, Bork P, et al. Systematic identification of genes with coding microsatellites mutated in DNA mismatch repair-deficient cancer cells. Int J Cancer. 2001;93:12-19.

41. Ta RM, Hecht JL, Lin DI. Discordant loss of mismatch repair proteins in advanced endometrial endometrioid carcinoma compared to paired primary uterine tumors. Gynecol Oncol. 2018;151:401-6.

42. Chapel DB, Lengyel E, Ritterhouse LL, Lastra RR. Interpretation of mismatch repair protein immunohistochemistry in endometrial carcinoma should consider both lynch syndrome screening and immunotherapy susceptibility: an illustrative case report. Int J Gynecol Pathol. 2019. https://doi.org/10.1097/PGP. 0000000000000594.

43. Simpkins SB, Bocker T, Swisher EM, Mutch DG, Gersell DJ, Kovatich AJ, et al. MLH1 promoter methylation and gene silencing is the primary cause of microsatellite instability in sporadic endometrial cancers. Hum Mol Genet. 1999;8:661-6. 